\title{
Avaliação da mineralização dos segundos molares inferiores como parâmetro para a classificação da idade biológica
}

\begin{abstract}
RESUMO
O presente trabalho teve como objetivo avaliar se a mineralização dos segundos molares inferiores permanentes pode ser usado como parâmetro para classificar a idade biológica do indivíduo. A amostra foi constituída por 129 radiografias panorâmicas, sendo 71 indivíduos do sexo feminino e 58 indivíduos do sexo masculino, na faixa etária de 7 anos à 12 anos e 1 mês. Para a análise da mineralização dental foi utilizada a tabela proposta por Nolla (1960). Os resultados da análise foram documentados numa planilha do programa Microsoft Excel 2010 contendo o nome completo, data de nascimento, data da tomada radiográfica, idade em anos e meses, número do prontuário, estágio de Nolla (1960) lado direito e lado esquerdo. Foi realizada a análise estatística (Mann-Whitney, Wilcoxon e correlação de Spearman) e pôde-se concluir que na amostra estudada não foi encontrado dimorfismo sexual, que a mineralização dentária ocorre de forma similar do lado direito e esquerdo, e que a mineralização dos segundos molares inferiores permanentes podem ser usadas como parâmetro para estimar a idade biológica e cronológica de um indivíduo.

Palavras-chave: Calcificação Fisiológica, Dente Molar, Análise Biológica.
\end{abstract}

\section{ABSTRACT}

This paper aimed to evaluate if the mineralization of permanent second molars can be used as a parameter to classify the biological age of the individual. The sample was composed of 129 panoramic radiographs, being 71 females and 58 males, aged 7 years and 12 years and 1 month. For the analysis of dental mineralization it was used a table proposed by Nolla (1960) with X-rays on the negatoscope (light box). The analysis results were documented in a Excel spreadsheet containing the full name, date of birth, date of the radiographic procedure, age in years and months, medical record number, stage of Nolla (1960) right and left side. Performed a statistical analysis, we concluded in our survey that the tooth mineralization occurs similarly in the right and left side, there is a certain precocity when compared to chronological age and the stage of mineralization in females compared to males. We also conclude that within the same chronological age, girls and boys have different mineralization stages, indicating that the dental mineralization can be used to identify the biological age, and the same is poorly correlated with chronological age.

Key-words: Calcification, Physiologic, Molar Tooth, Biological Analysis.

\section{Gisele do Amaral Móra*}

Nádia Altobello Abatayguara

Adametes**

Kurt Faltin Junior ${ }^{* * *}$

Cristina Lúcia Feijó Ortolani****

Roberto H. Matsui*****

* Graduada em Odontologia - UNIP

** Mestra em Ortodontia e doutoranda em Ortodontia - UNIP

*** Doutor em Ortodontia - Universidade de Bonn, Alemanha

**** Doutora em Diagnóstico Bucal - USP

*****Doutor em Biopatologia Bucal - UNESP

Submetido em: 16-5-2017

Aceito em: 26-7-2017 


\section{INTRODUÇÃO}

A estimativa da idade biológica é de grande utilidade na Odontologia, já que a idade cronológica sozinha, nem sempre é um bom parâmetro para avaliar o estágio da maturação em que o corpo se encontra.

A idade cronológica é baseada a partir do dia do nascimento e exprime a quantidade de tempo de vida de um indivíduo. Possui efeito documental e legal. Esta é sem dúvidas a mais utilizada dentro da sociedade. E os métodos de identificação da idade biológica também auxiliam em processos criminais, legais e de perícia, se estimando a provável idade cronológica ${ }^{1}$.

A expressão "idade biológica" é fortemente associada à idade óssea, mas o termo idade biológica refere-se além dos aspectos de maturação óssea, envolvendo também aspectos morfológicos, dentários, mentais e circumpuberais ${ }^{2}$.

A idade mental é estimada através da maturidade psicológica do indivíduo e envolve estágios de desenvolvimento emocional e de capacidades intelectuais.

A idade morfológica relaciona-se com a maturidade biológica através da estatura e forma. A altura e o peso fazem parte do crescimento geral somático, processo que se inicia na concepção e persiste até o final da adolescência e apesar de ocorrer em todos os indivíduos, há variação de intensidade e duração e sofrem influências de fatores genéticos e ambientais. Depois de atingir sua estatura, indivíduos da mesma idade poderão ter pesos e altura diferentes. O primeiro período de estirão ocorre de 0 a 2 anos de idade.

A idade esquelética é observada através da avaliação do estágio de desenvolvimento que se encontram os diferentes centros de ossificação do corpo, como por exemplo, o pé, ombro, cotovelo, joelho, mão, crânio, vértebras cervicais quadril e pelve, entre outros ${ }^{2}$.

Dentre os métodos mais pesquisados relacionados ao crescimento facial temos o desenvolvimento da mão e punho, pico de crescimento puberal, estágio de maturação das vértebras cervicais e mineralização dentária ${ }^{1}$.

A região de mão e punho foi por muito tempo na ortodontia a mais utilizada por possuir um grande número de centro de ossificação e por apresentar três tipos de ossos longos, curtos e arredondados e com uma radiografia simples e de baixo custo podia-se avaliar o crescimento ósseo do paciente a fim de analisar qual o estágio de maturação ele se encontrava ${ }^{3}$.

Hoje são mais utilizados pelos ortodontistas os indicadores de maturação das vértebras cervicais proposta por Hassel \& Farman ${ }^{3}$, esta é analisada através de teleradiografias em norma lateral e também mostra em qual processo de desenvolvimento o paciente se encontra, mostrando o momento mais oportuno para promover crescimento dos maxilares.

O surto de crescimento puberal (SCP) é o segundo período de estirão. Na menina ocorre mais precocemente, entre 9 e 11 anos, e envolve desenvolvimento do tecido mamário, pubarca e desacelera após a menarca. No menino ocorre entre 11 a 13 anos, e envolve volume testicular, pubarca, forma genital, mudança na voz e pelos faciais. Este período é sem dúvidas a fase mais favorável para se estimular e direcionar o crescimento da face ${ }^{1}$.

A cronologia de erupção também é um método usado para se estimar a idade biológica de um indivíduo, mas este pode não ser tão confiável, pois pode haver extrações precoces ou tardias de dentes decíduos, patologias diversas e alguns dentes podem ficar 
impactados e ou então haver a anadontia de algum elemento e esses fatores podem alterar a sequência e a época de erupção e ou o dente não irromper a cavidade bucal. Diversos artigos demonstraram haver estatisticamente alta correlação entre a maturação dentária, maturação óssea e idade cronológica em ambos os sexos ${ }^{4,5,6}$.

Enquanto alguns autores acreditam na correlação entre maturação óssea e a mineralização dentária ${ }^{1,7-16}$ outros descordam ${ }^{2,17-22}$. Desta forma, foi realizada esta pesquisa sobre a mineralização dos segundo molares permanentes inferiores a fim de analisarmos se este pode ser usado como método auxiliar para classificar a idade biológica do indivíduo.

\section{PROPOSIÇÃO}

- Identificar se os estágios de mineralização dos segundos molares permanentes inferiores podem ser usados como parâmetro para classificar a idade biológica do indivíduo. - Avaliar se há dimorfismo sexual quanto à mineralização dos dentes 37 e 47.

- Verificar se existe semelhança no desenvolvimento em dentes homólogos

\section{MATERIAL E MÉTODO}

Com a aprovação pelo Comitê de Ética em Pesquisa da Universalidade Paulista (UNIP) de São Paulo, sob o protocolo número 142.528 (em anexo), iniciou-se o estudo.

A partir de 1000 pantomografias da Clínica Infantil do Departamento de Odontologia do Instituto de Ciências da Saúde da Universidade Paulista (UNIP), situada na cidade de São Paulo foram selecionadas um total de 129 radiografias panorâmicas, sendo 71 números de amostras femininas de idades entre 7 anos e 2 meses à 11 anos e 10 meses e 58 amostras masculinas de idades entre 7 anos e 1 mês à 12 anos e 1 mês.

Os critérios de seleção das amostras foram utilizar radiografias que estivessem em bom estado, possibilitando a análise e foram estipuladas as idades entre 7 anos à 12 anos e 1 mês.

As tomadas radiográficas foram obtidas pelo método e técnica convencional de radiografia panorâmica.

Foram selecionadas primeiramente 45 radiografias femininas e 40 radiografias masculinas. Foram feitas as análises e colocado o resultado numa tabela do programa Microsoft Excel 2010. Depois estas radiografias foram escaneadas no aparelho marca HPSCANJET-G 4050 e gravada em CD-ROM e então foi feita uma nova análise através das imagens no computador e os resultados obtidos foram colocados na mesma tabela, posteriormente foi selecionado mais 26 radiografias femininas e 18 radiografias masculinas seguindo o mesmo padrão de inclusão. Estas foram avaliadas as duas vezes somente pelo método visual e os resultados colocados numa outra tabela do programa Microsoft Excel 2010.

As radiografias panorâmicas foram colocadas sobre o negatoscópio da marca Softline e avaliadas numa sala escura. As imagens radiográficas foram analisadas por 2 examinadores numa quantidade de 20 radiografias por dia para evitar fadiga visual. E após 1 mês, período este suficiente para que os examinadores não se recordassem das avaliações, as imagens foram reavaliadas. 
Para a avaliação do desenvolvimento dentário utilizou-se as radiografias panorâmicas. Foram aplicadas as 10 fases da mineralização proposta por Nolla (1960) sendo elas: estágio 0: ausência de cripta; estágio 1: presença de cripta, estágio 2: calcificação inicial da coroa; estágio 3: 1/3 da coroa completa; estágio 4: 2/3 da coroa completa; estágio 5: coroa praticamente completa; estágio 6: coroa completa; estágio 7: 1/3 da raiz completa; estágio 8: 2/3 da raiz completa; estágio 9: raiz praticamente completa mas com o ápice aberto; estágio 10: raiz completa e ápice fechado.

FIGURA 1 - Representação esquemática dos 10 estágios de desenvolvimento dentário segundo Nolla (1960) (Almeida 2009).

\section{DENTES INFERIORES}

Estágio 0

Estágio ।

Estágio 2

Estágio 3

Estágio 4

Estágio 5

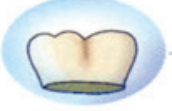

Estágio 6

Estágio 7

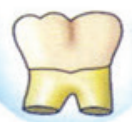

Estágio 8

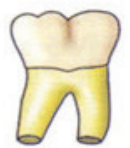

Estágio 9

Estágio 10
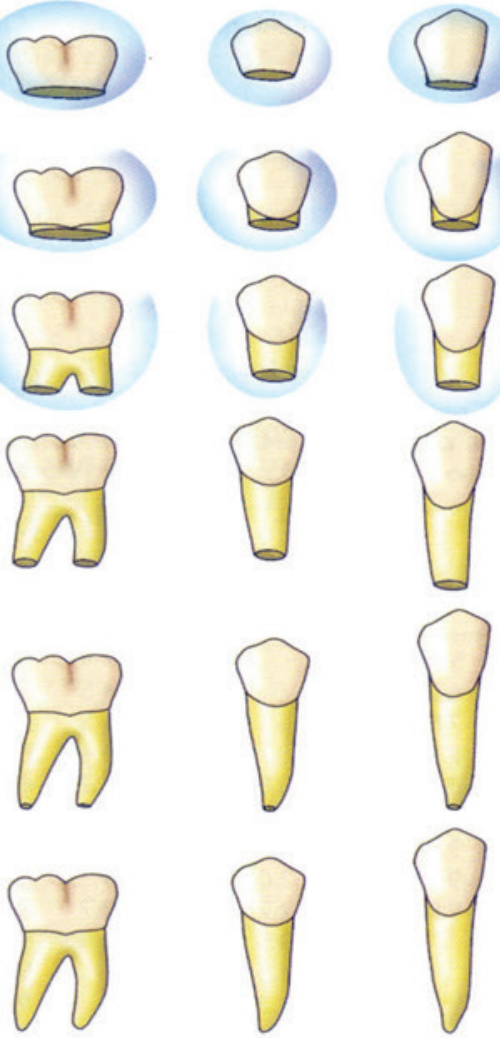

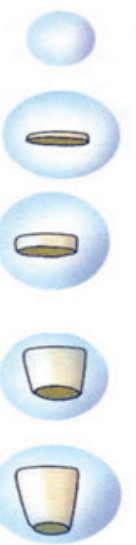

presença de cripta

calcificação inicial da coroa

1/3 da coroa completa

$2 / 3$ da coroa completa

coroa praticamente completa

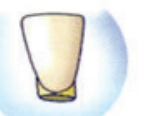

coroa completa

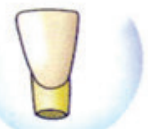

1/3 da raiz completa

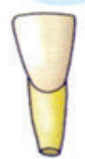

2/3 da raiz completa

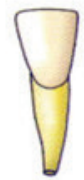

raiz praticamente completa mas com ápice aberto

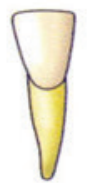

raiz completa e ápice fechado. 
Foram avaliados os dentes segundo molares permanentes inferiores do lado direito e esquerdo.

Figura 2 - Observação dos segundos molares inferiores permanentes direito e esquerdo através de radiografia panorâmica.

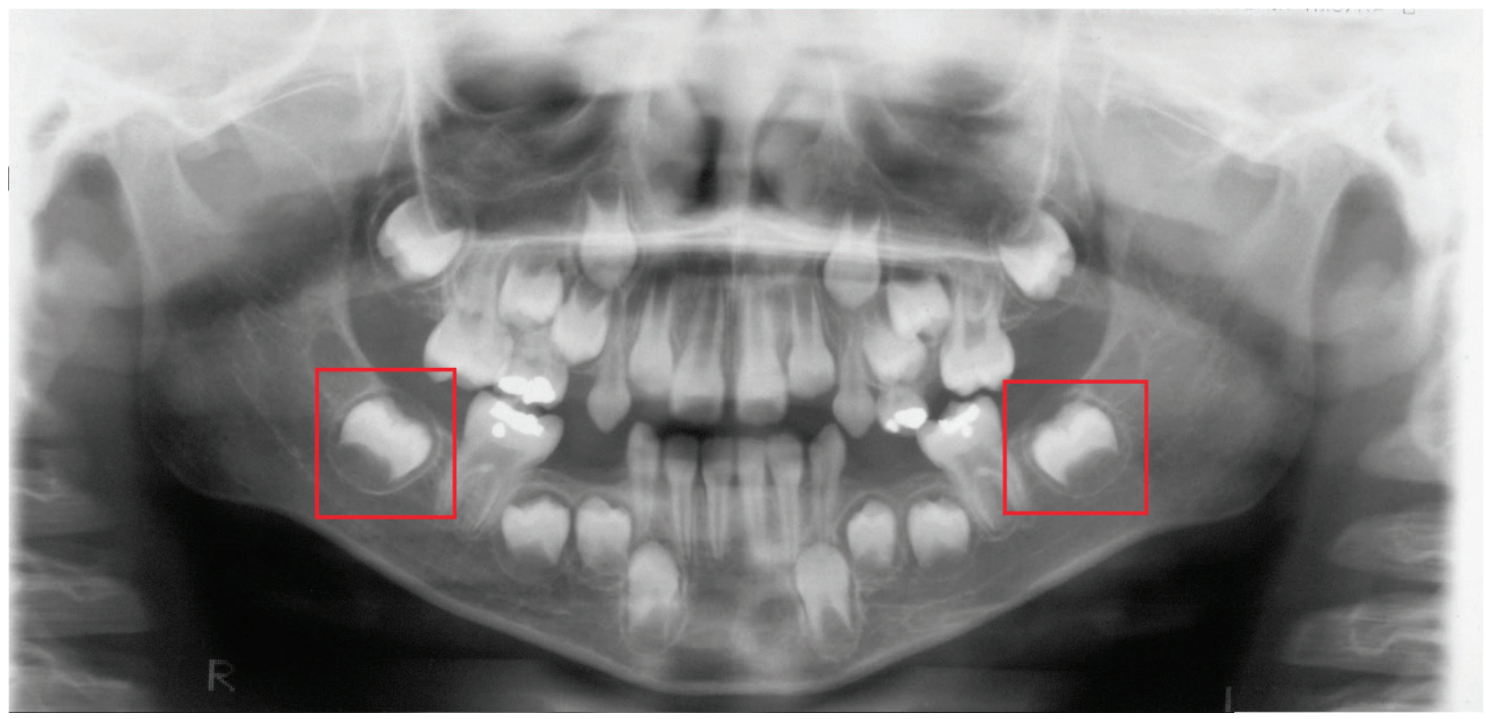

\section{CONSTRUÇÃO DA TABELA}

Foi verificada a fase em que cada dente se encontrava e buscou-se na tabela de Nolla. Os dados foram anotados em tabela do programa do Microsoft Excel 2010 separados por sexo e em ordem da idade cronológica em anos e meses, contendo: nome completo, número do prontuário, data do nascimento, data da tomada radiográfica, respectiva idade cronológica no momento da tomada radiográfica e o estágio de mineralização que o dente se encontrava do lado direito e esquerdo da primeira e segunda análise.

Nos casos de dúvidas em definir o estágio exato do desenvolvimento dentário, optou-se por classificar o dente pelo menor estágio.

\section{ANÁLISE ESTATÍSTICA}

Foi realizado o Teste de Normalidade de Anderson-Darling e optou-se pela utilização de testes de comparação não paramétricos. Então foi feito o teste U de Mann-Whitney para avaliar se houve dimorfismo sexual. O teste $U$ de Wilcoxon para realizar a comparação da mineralização dos molares inferiores direito e esquerdo. O teste de correlação de Spearman para verificar se a mineralização dos segundos molares inferiores podem ser usados como parâmetro para classificação da idade biológica.

\section{RESULTADOS}

Foi realizado primeiramente o teste de Normalidade de Anderson-Darling, sendo: H0 (Hipótese Nula): Os dados seguem a distribuição Normal. 
H1 (Hipótese Alternativa): Os dados não seguem a distribuição Normal.

Obteve-se um Nível de significância de 0,05.

E como todas as amostras apresentaram um valor de $\mathrm{p}$ menor que o nível de significância; nenhuma delas segue a distribuição Normal, portanto optou-se pela utilização de testes de comparação não-paramétricos.

\begin{tabular}{|l|l|l|l|}
\hline & Total & Feminino & Masculino \\
\hline Nolla direito & \multicolumn{1}{|c|}{$3,886 \mathrm{E}-12$} & $1,0315 \mathrm{E}-09$ & $3,2557 \mathrm{E}-05$ \\
\hline Nolla esquerdo & $2,9565 \mathrm{E}-12$ & $1,6695 \mathrm{E}-09$ & $1,3156 \mathrm{E}-05$ \\
\hline Análise direito & $2,7202 \mathrm{E}-15$ & $3,2937 \mathrm{E}-10$ & $5,538 \mathrm{E}-06$ \\
\hline Análise esquerdo & $2,044 \mathrm{E}-15$ & $1,3165 \mathrm{E}-10$ & $3,949 \mathrm{E}-06$ \\
\hline
\end{tabular}

Para verificar o dimorfismo sexual quanto a maturação dos respectivos dentes, foi realizado o Teste U de Mann-Whitney (Teste não-paramétrico de comparação de 2 amostras independentes) sendo:

H0 (Hipótese Nula): Não há diferença entre a amostra do sexo feminino quando comparada com a amostra do sexo masculino.

H1 (Hipótese Alternativa): Há diferença entre a amostra do sexo feminino quando comparada com a amostra do sexo masculino.

Nível de significância: 0,05.

\begin{tabular}{|l|l|}
\hline & Valor de $\mathrm{p}$ \\
\hline Nolla direito & 0.2633073 \\
\hline Nolla esquerdo & 0.3144382 \\
\hline Análise direito & 0.1239370 \\
\hline Análise esquerdo & 0.1294436 \\
\hline
\end{tabular}

Como todas as amostras apresentaram um valor de $\mathrm{p}$ maior que o nível de significância, não se rejeita H0, e pode-se dizer que não há dimorfismo sexual quanto a maturação dos respectivos dentes.

\section{SIMETRIA DA MINERALIZAÇÃO DO LADO DIREITO E ESQUERDO}

Para comparar o grau de calcificação dos segundo molar inferior direito com o segundo molar inferior esquerdo foi realizado o teste $U$ de Wilcoxon (Teste não-paramétrico de comparação de 2 amostras dependentes), sendo:

H0 (Hipótese Nula): Não há diferença no grau de calcificação das amostras do lado direito e esquerdo.

H1 (Hipótese Alternativa): Há diferença no grau de calcificação das amostras do lado direito e esquerdo.

Nível de significância: 0,05.

\begin{tabular}{|l|l|l|l|}
\hline Valores de $\mathrm{p}$ & Total & Feminino & Masculino \\
\hline Nolla direito vs esquerdo & 0.6547208 & 0.3173105 & 0.3173105 \\
\hline Análise direito vs esquerdo & 1 & 0.6547208 & 0.3173105 \\
\hline
\end{tabular}


Como todas as amostras apresentaram um valor de $\mathrm{p}$ maior que o nível de significância, não se rejeita H0, e pode-se dizer que não há diferença no grau de calcificação dos segundo molar inferior direito com o segundo molar inferior esquerdo, independentemente do grupo que originou a amostra.

Para verificar se os estágios da mineralização dos segundos molares inferiores podem ser considerados como parâmetro para avaliação da idade biológica foi realizado o teste de Correlação de Spearman (Teste de correlação não-paramétrico), sendo:

H0 (Hipótese Nula): Não há correlação significativa entre os estágios de mineralização dos segundos molares inferiores e a idade biológica.

H1 (Hipótese Alternativa): Há correlação significativa entre os estágios de mineralização dos segundos molares inferiores e a idade biológica.

Nível de significância: 0,05.

\begin{tabular}{|l|l|l|}
\hline & Total rho & Valor de $\mathrm{p}$ \\
\hline Nolla direito & 0.6318744 & $0.0000000000000007545043^{*}$ \\
\hline Nolla esquerdo & 0.6327555 & $0.0000000000000006690764^{*}$ \\
\hline Análise direito & 0.6284694 & $0.0000000000000011961166^{*}$ \\
\hline Análise esquerdo & 0.6303829 & $0.0000000000000009238853^{*}$ \\
\hline
\end{tabular}

${ }^{*}$ Correlação significativa

\begin{tabular}{|l|l|l|}
\hline & Feminino rho & Valor de p \\
\hline Nolla direito & 0.6676976 & $0.000000000201841216^{*}$ \\
\hline Nolla esquerdo & 0.6658564 & $0.000000000235708640^{*}$ \\
\hline Análise direito & 0.7017424 & $0.000000000009287767^{*}$ \\
\hline Análise esquerdo & 0.7029058 & $0.000000000008296848^{*}$ \\
\hline
\end{tabular}

*Correlação significativa

\begin{tabular}{|l|l|l|}
\hline & Masculino rho & Valor de $\mathrm{p}$ \\
\hline Nolla direito & 0.5871711 & $0.0000010174616^{*}$ \\
\hline Nolla esquerdo & 0.5915602 & $0.0000008061988^{*}$ \\
\hline Análise direito & 0.5687883 & $0.0000026010810^{*}$ \\
\hline Análise esquerdo & 0.5756197 & $0.0000018473895^{*}$ \\
\hline
\end{tabular}

${ }^{*}$ Correlação significativa

Como todas as amostras apresentaram um valor de p menor que o nível de significância, rejeita-se H0, e pode-se dizer que há correlação significativa entre os estágios de mineralização dos segundos molares inferiores com a idade biológica. Além disso, como em todos os casos o coeficiente de correlação de Spearman rho encontrado foi maior que 0 , têm-se que as correlações encontradas foram positivas, ou seja, à medida que a idade aumenta os estágios de mineralização dos segundos molares inferiores também aumentam.

\section{DISCUSS ÃO}

A Idade biológica é aquela que melhor representa o estágio de formação do indivíduo, dentro de uma idade cronológica há a possibilidade de haver inúmeros estágios de crescimento e são esses estágios de maturação que determinam a idade biológica da pessoa.

Fatores importantes são utilizados como parâmetros para acompanhar o crescimento e desenvolvimento de cada indivíduo, como exemplo o surto de crescimento puberal, 
a idade óssea, a maturação das vértebras cervicais e a sequência de erupção dos dentes, entre outros, visto que o processo de maturação ocorre em velocidades e estágios diferentes para cada pessoa.

Através dos exames complementares o ortodontista pode estimar em qual fase de crescimento seu paciente se encontra e realizar um diagnóstico e plano de tratamento mais adequado para cada caso. A radiografia panorâmica possibilita observar uma série de fatores importantes como a existência ou não dos germes dentais, avaliar a simetria do lado direito e esquerdo, presença de patologias e estruturas existentes.

Assim como os trabalhos de Peter 2003, Eto e Mazzieiro 2005, Kohatsu 2008, Martins 2009 e Vieira et al. 2009, foi utilizada a classificação desenvolvida por Nolla (1960) por ser a mais citada dentre os anos e a mais usada internacionalmente.

Já outros trabalhos como de Uysal 2004, Santana 2007, Almeida 2009, Araujo et al. 2010 e Ogodescu et al. 2011, utilizaram o método de avaliação preconizado por Demirjian.

E outros autores como Saliba 2005, Manhães 2006, Camargo e Cunha 2007, Duarte et al. 2008, Kohatsu 2008, Oliveira et al 2010 e Gonçalves e Antunes 1999, fizeram a análise preconizada por Nicodemo et al.

Foram utilizados os dentes segundo molares inferiores, por sua visualização ser mais fácil na mandíbula, sem sofrer sobreposição de imagens de estruturas adjacentes como ocorre na maxila. E também por já haver trabalhos dizendo que há certa correlação da mineralização desses dentes com outros parâmetros de crescimento. (Peter, 2003; Manhães, 2007; Santana, 2007; Martins, 2009; Vieira, 2009; Gonçalves e Antunes, 1999; Moraes, 1998).

É importante ressaltar que buscar métodos dentro do arsenal de documentos já necessários para o plano de tratamento ortodôntico, dispensa a necessidade de se realizar exames adicionais para a determinação da idade biológica, evitando mais exposição radiográfica e minimizando custos. Por esta razão é de grande interesse poder concluir que a mineralização dos segundos molares inferiores podem e devem ser usado como um dos métodos para determinar a idade biológica da criança e adolescente, pois esta análise é feita facilmente pela radiografia panorâmica.

Em nossa amostra, estatisticamente não houve dimorfismo sexual em relação a mineralização dos $2^{\circ}$ molares inferiores permanentes. Mas esse resultado pode ter ocorrido devido a nossa amostra, pois é sabido que as meninas possuem um processo de desenvolvimento mais acelerado quando comparado a meninos da mesma idade cronológica, assim como mostra os trabalhos de Almeida 2009, Santana 2007, Vieira 2009 e Ogodescu 2011.

Nossos resultados concordam com Duarte (2008) e Peter (2013), na qual encontraram que à medida que a idade aumenta, a mineralização dentária também aumenta, havendo, portanto, significativa correlação entre mineralização dentária e idade cronológica e difere do trabalho de Camargo e Cunha (2007) que não observou sincronismo em relação à maturação dental com a idade cronológica, dizendo apenas haver uma próxima relação entre ambas, diminuindo a confiabilidade desse método.

Santana (2007), Almeida (2009), Vieira (2009) e Ogodescu (2011), em suas amostras encontraram no sexo feminino uma tendência ao desenvolvimento dentário mais precoce em reação ao sexo masculino, diferente do que encontramos dentro do nosso grupo estudado.

Nossos resultados concordam com os trabalhos de Camargo e Cunha (2007), Kohatsu (2008) e Araujo (2010), na qual não encontraram diferença significativa estatisticamente entre o sexo feminino e masculino em relação à mineralização dentária. 
Não encontramos diferença de mineralização do segundo molar inferior do lado direito e esquerdo, assim como no trabalho de Kohatsu (2008) e Almeida (2009) e Araujo (2010).

Nossos resultados discordam de Benemann (1997) que afirma que a idade dentária possui grandes riscos a alterações ambientais, não sendo adequada para estabelecer a idade biológica, pois em nossa pesquisa podemos observar que a mineralização dental acompanha a idade cronológica de forma significativa, acompanhando por tanto a respectiva idade biológica.

\section{CONSIDERAÇÕES FINAIS}

Concluímos com o nosso trabalho que os estágios da mineralização dos segundos molares inferiores permanentes podem ser usados como mais um parâmetro para avaliar a idade biológica, com correlação significativa em relação à idade cronológica.

Não foi possível observar dimorfismo sexual na amostra estuda e a mineralização dos segundos molares inferiores direito e esquerdo ocorrem simultaneamente.

\section{REFERÊNCIAS}

1. Gonçalves, ACS; Antunes, JLF. Estimativa da Idade em Crianças Baseada nos Estágios de Mineralização dos Dentes Permanentes, com Finalidade Odontolegal. São Paulo: Odontologia e Sociedade, v.1, n.1/2, 1999.

2. Benemann, E; Sampaio, R; Berthold, T. Idade biológica: Indicadores do surto de crescimento. Ortod Gaúcha, v.1, n.1, abr. 1997.

3. Hassel B, Farman AG. Skeletal maturation evaluation using cervical vertebrae. Am J Orthod Dentofacial Orthop 1995 Jun;107(6):19.

4. Peter, EA. Relação entre surto de crescimento puberal, desenvolvimento dentário e idade cronológica em crianças e adolescentes na faixa etária de 7 a 15 anos. 2003. 132 folhas. Mestrado - Universidade de São Paulo, Bauru, 2003.

5. Uysal, T. et al. Relationships between dental and skeletal maturity in Turkish subjects. Angle Orthod, v.74, n.5, Oct. 2004. 657-64 p.

6. Camargo, GTL; Cunha, TGE. Estudo do Sincronismo entre o índice de maturação das vertebras cervicais, idade dentária e idade carpal com a idade cronológica. SOTAU - Revista virtual de odontologia, v. 2, 2007. Disponível em <sotau.sind.googlepages.com/revista>. Acessado em 02 jun. 2013.

7. Santana, VC. Comparação entre maturação das vértebras cervicais e desenvolvimento dentário de caninos e segundo molares mandibulares em indivíduos do sexo masculino e feminino na faixa etária de 8 a 13,4 anos. 2007. 130 folhas. Mestrado Universidade Paulista - São Paulo, 2007.

8. Kohatsu, LI. Estudo radiográfico da relação entre os principais métodos de avaliação da idade óssea e dentária com a idade cronológica quando aplicados à leucodermas e xantodermas brasileiros. 2008. 128 folhas. Doutorado - Universidade Estadual Paulista, São José dos Campos 2008.

9. Almeida, M. Correlação entre a mineralização dos segundos molares permanentes e idade cronológica numa amostra populacional de Paraíba. 2009. 59 folhas. Monografia. Universidade Federal Da Paraíba, João Pessoa, 2009.

10. Carvalho, TAA. Mineralização dentária na estimativa da idade do indivíduo. 2009. 54 folhas. Monografia. Universidade Federal da Paraíba, João Pessoa, 2009.

11. Martins, AL. Relação entre métodos de avaliação do surto de crescimento puberal e os estágios de mineralização dentária, e sua aplicação clínica. 2009. 94 folhas. Doutorado - Universidade Federal de Mato Grosso do Sul, Campo Grande, 2009.

12. Vieira, CL. et al. Relação entre os índices de maturação das vértebras cervicais e os estágios de calcificação dentária. Maringá, R Dental Press Ortodon Ortop Facial, v. 14, n. 2, mar./abr. 2009. 45-53 p.

13. Araújo, A. et al. Association between mineralization of third molars and chronological age in a Brazilian sample. Rev. odonto ciênc.n.25,v.4. 2010.

14. Oliveira, OF. et al. Estimativa da idade por meio de radiografias panorâmicas. RGO - Rev Gaúcha Odontol., Porto Alegre, n. 2, v. 58, abr./jun. 2010. 203-206 p. 
15. Ogodescu, AE. Estimation of child's biological age based on tooth development. Romanian Jornal of Legal Medicine, v.19, n.2, 2011.

16. Moraes, MEL; Medici Filho, E; Moraes, LC. Surto de Crescimento Puberal. Relação entre Mineralização Dentária, Idade Cronológica, Idade Óssea - Método Radiográfico. São Paulo: Revista Odontol. UNESP, n.27, v.1, 1998.

17. Duarte, HEM. et al. Avaliação das idades dentária e óssea em indivíduos situados antes do surto de crescimento puberal. OrtodontiaSPO, n.41, v.2. 2008.

18. Eto, LF. MAZZIEIRO, Ênio Tonani. Avaliação da correlação entre os estágios de mineralização dos dentes inferiores e a idade esquelética observados sob o gráfico do crescimento puberal. R Dental Press Ortodon Ortop Facial. Maringá, v.10, n.2, mar./abr. 2005, 75-86 p.

19. Saliba, JHM. Estudo radiográfico comparativo entre a cronologia de mineralização dentária e a maturação óssea das vértebras cervicais. 2005. 83 folhas. Mestrado - Universidade Vale do Rio Verde, Três Corações, 2005.

20. Bernal, N; Arias, MI. Indicadores de maduracion esqueletica y dental. Revista CES Odontología, v. 20, n.1, 2007.

21. Ré, AHN. Crescimento, maturação e desenvolvimento na infância e adolescência: Implicações para o esporte. Motricidade, v.7 n.3. 2011, 55-67 p.

22. Hilgenberg, SP; Pinto, SCS; Pinheiro, JC. Comparação entre as idades óssea, dentária e cronológica por meio de método radiográfico simplificado. Rev Odonto, n.32, jul/dez. 2008, 31-38 p.

23. Júnior, LRCM. et al. Correlação entre a maturação óssea das vértebras cervicais com a maturação óssea de mão e punho e com a mineralização dentária pelo método radiográfico. OrtodontiaSPO, n.4, v.4, Out/ Dez. 2006. 